\title{
Burden of Palliative Care Issues Encountered by Radiation Oncologists Caring for Patients With Advanced Cancer
}

\section{Citation}

Parker, Gregory. 2017. Burden of Palliative Care Issues Encountered by Radiation Oncologists Caring for Patients With Advanced Cancer. Doctoral dissertation, Harvard Medical School.

\section{Permanent link}

http://nrs.harvard.edu/urn-3:HUL.InstRepos:40621395

\section{Terms of Use}

This article was downloaded from Harvard University's DASH repository, and is made available under the terms and conditions applicable to Other Posted Material, as set forth at http:// nrs.harvard.edu/urn-3:HUL.InstRepos:dash.current.terms-of-use\#LAA

\section{Share Your Story}

The Harvard community has made this article openly available.

Please share how this access benefits you. Submit a story.

\section{Accessibility}


Scholarly Report submitted in partial fulfillment of the MD Degree at Harvard Medical School

Date: 30 January 2017

Student Name: Gregory Parker, BS

Scholarly Report Title: Burden of palliative care issues encountered by radiation oncologists caring for patients with advanced cancer

Mentor Name and Affiliations: Tracy Balboni, MD, MPH; Department of Radiation Oncology, Dana-Farber/Brigham and Women's Cancer Center; Department of Psychosocial Oncology and Palliative Care, Dana-Farber Cancer Institute

Collaborators: Virginia T. LeBaron, PhD, APRN, FAANP ${ }^{2}$; Monica Krishnan, $\mathrm{MD}^{1,3}$; Ron Y. Shiloh, $\mathrm{MD}^{3}$; Alexander Spektor, MD, $\mathrm{PhD}^{1,3}$; Lauren Hertan, MD, $\mathrm{MS}^{3}$

${ }^{1}$ Harvard Medical School, Boston, MA

${ }^{2}$ University of Virginia, School of Nursing, Charlottesville, VA

${ }^{3}$ Department of Radiation Oncology, Dana-Farber/Brigham and Women's Cancer Center, Boston, MA 


\section{Abstract \\ TITLE: Burden of palliative care issues encountered by radiation oncologists caring for patients with advanced cancer}

Gregory M. Parker, Virginia T. LeBaron, Monica Krishnan, Ron Y. Shiloh, Alexander Spektor, Lauren Hertan, Tracy A. Balboni

Purpose: Radiation oncologists frequently provide care for patients with advanced cancer who are in their last months or weeks of life. This study examined the previously not well characterized types and frequencies of palliative care issues encountered in consultations for palliative radiation therapy (PRT).

Methods: This prospective, survey-based study assessed consecutive consults for PRT from 5/19/14 to 9/26/14 at three Boston-area, community and academic, hospital-based centers. Participating physicians and nurse practitioners completed a survey to identify and rank the relevance (5-point Likert scale, 'not at all' to 'extremely') of palliative care issues. Eight domains adapted from national palliative care guidelines - physical symptoms, psychosocial issues, cultural considerations, spiritual needs, care coordination, advance care planning, goals of care, and ethical and legal issues - were evaluated. 162 consecutive consultations were surveyed with 140 responses received ( $86 \%$ response rate).

Results: Most ( $82 \%$ ) consults had two or more palliative care domains ranked as highly ('very' or 'extremely') relevant to care. The domains of physical symptoms (91\%), care coordination $(70 \%)$, goals of care $(59 \%)$, and psychosocial issues $(52 \%)$ were the most commonly reported domains as highly relevant to care. Forty-six percent of consults involved a high palliative care burden (four or more palliative care domains identified as highly relevant to care). Predictors of high palliative care burden in multivariable analysis were ECOG performance status $>2$ $(\mathrm{OR}=3.57, \mathrm{p}=0.047)$, a plan for no further anti-cancer therapy after PRT (OR 3.46, p = 0.03), and a recommendation against PRT $(\mathrm{OR} 4.80, \mathrm{p}=0.01)$.

Conclusions: Radiation oncology clinicians encounter multiple palliative care issues when consulting on patients for PRT. Clinicians identified physical symptoms, care coordination, and goals of care as the most relevant palliative care domains. These findings can help guide palliative care development within radiation oncology, including education and structures of care delivery. 


\section{Summary of Personal Contribution to the Scholarly Project}

In fulfillment of the Scholarly Project requirement I completed an original research study under the mentorship of Dr. Tracy Balboni from the Deparment of Radiation Oncology at BWH. The title of my work was "Burden of palliative care issues encountered by radiation oncologists caring for patients with advanced cancer."

I served as the lead investigator on this study. In regards to the study design I developed this in partnership with Dr. Balboni and with suggestions from the co-authors of the study. I performed the necessary literature review that formed the background for the study and is summarized in the accompanying manuscript. The survey instrument used in this study was drafted by myself and then reviewed and edited by Dr. Balboni and the other co-authors.

I oversaw the data collection process as outlined in the accompanying manuscript. I created the database into which all original data was entered. I performed the initial data analysis including the creation of the manuscripts tables and figures. Dr. Balboni and I worked together to perform the statistical analyses that are referenced in the manuscript.

I drafted the entire manuscript and managed the review process among the co-authors. I was first author on the submitted manuscript. 
Appendix 1. Complete preprint version of submitted manuscript to the journal, Practical Radiation Oncology (PRO).

\section{TITLE: Burden of palliative care issues encountered by radiation oncologists caring for patients with advanced cancer}

Gregory M. Parker, BS ${ }^{1}$; Virginia T. LeBaron, PhD, APRN, FAANP ${ }^{2}$; Monica Krishnan, MD ${ }^{1,3}$;

Ron Y. Shiloh, $\mathrm{MD}^{3}$; Alexander Spektor, MD, $\mathrm{PhD}^{1,3}$; Lauren Hertan, MD, $\mathrm{MS}^{3}$; Tracy A. Balboni, MD, MPH ${ }^{1,3,4}$

${ }^{1}$ Harvard Medical School, Boston, MA

${ }^{2}$ University of Virginia, School of Nursing, Charlottesville, VA

${ }^{3}$ Department of Radiation Oncology, Dana-Farber/Brigham and Women's Cancer Center, Boston, MA

${ }^{4}$ Department of Psychosocial Oncology and Palliative Care, Dana-Farber Cancer Institute, Boston, MA

Corresponding Author: Tracy Balboni; Department of Radiation Oncology, Dana-Farber Cancer Institute, 450 Brookline Ave, Boston, MA 02215; 617-378-5009;

TBALBONI@BWH.HARVARD.EDU

RUNNING HEAD: Palliative Care Issues in Radiation Oncology

PRIOR PRESENTATIONS: Palliative Care in Oncology Symposium, 2015 Annual Assembly Abstract Presentation

CONFLICTS OF INTEREST NOTIFICATION: The authors have no actual or potential conflicts of interest to report.

FUNDING SOURCES: Grant from Harvard Medical School Scholars in Medicine Office 


\begin{abstract}
Purpose: Radiation oncologists frequently provide care for patients with advanced cancer who are in their last months or weeks of life. This study examined the previously not well characterized types and frequencies of palliative care issues encountered in consultations for palliative radiation therapy (PRT).
\end{abstract}

Methods: This prospective, survey-based study assessed consecutive consults for PRT from 5/19/14 to 9/26/14 at three Boston-area, community and academic, hospital-based centers. Participating physicians and nurse practitioners completed a survey to identify and rank the relevance (5-point Likert scale, 'not at all' to 'extremely') of palliative care issues. Eight domains adapted from national palliative care guidelines - physical symptoms, psychosocial issues, cultural considerations, spiritual needs, care coordination, advance care planning, goals of care, and ethical and legal issues - were evaluated. 162 consecutive consultations were surveyed with 140 responses received ( $86 \%$ response rate).

Results: Most (82\%) consults had two or more palliative care domains ranked as highly ('very' or 'extremely') relevant to care. The domains of physical symptoms (91\%), care coordination (70\%), goals of care (59\%), and psychosocial issues (52\%) were the most commonly reported domains as highly relevant to care. Forty-six percent of consults involved a high palliative care burden (four or more palliative care domains identified as highly relevant to care). Predictors of high palliative care burden in multivariable analysis were ECOG performance status $>2$ $(\mathrm{OR}=3.57, \mathrm{p}=0.047)$, a plan for no further anti-cancer therapy after PRT (OR 3.46, p = 0.03), and a recommendation against PRT $(\mathrm{OR} 4.80, \mathrm{p}=0.01)$.

Conclusions: Radiation oncology clinicians encounter multiple palliative care issues when consulting on patients for PRT. Clinicians identified physical symptoms, care coordination, and goals of care as the most relevant palliative care domains. These findings can help guide palliative care development within radiation oncology, including education and structures of care delivery. 


\section{Introduction}

Radiation therapy is frequently administered with palliative intent in patients with advanced cancer. Over a 35-year period at the University of Texas M.D. Anderson Cancer center, 35 percent of patients treated in their department received palliative-intent radiation therapy. ${ }^{1}$ Even in the most recent year studied - 1995 - the proportion of patients treated with palliative-intent radiation therapy (PRT) remained unchanged at 34 percent. ${ }^{1}$ In a 2013 survey study, radiation oncologists estimated that 29 percent of their consultations were for palliative intent radiation therapy. ${ }^{2}$ Among patients with metastatic cancer, 30 percent receive radiation therapy in their last year of life, ${ }^{3}$ and of those receiving radiation therapy in the final month of life 18 percent spent 10 or more of their last 30 days receiving radiation treatments. ${ }^{4}$

Despite frequently encountering patients who are in the last year or months of their life, the field of radiation oncology has had little academic emphasis on palliative care, including a paucity of education and research in this area. ${ }^{1,5,6}$ Radiation oncologists are less likely than noncancer specialists to engage their terminally-ill patients in end-of-life discussions. ${ }^{7}$ Meanwhile, there is a consensus building that palliative care should be integrated into all stages of cancer care. ${ }^{8,9,10}$ In its 2013 report, Delivering High-Quality Cancer Care, the Institute of Medicine emphasized addressing patients' palliative care needs at all points along the cancer care continuum. ${ }^{8}$ In addition, the American Society of Clinical Oncologists describes a need for evidence-based design of palliative care training for practicing oncologists, residents, and fellows. ${ }^{9}$

While radiation therapy has long been used with palliative intent, there are limited data characterizing the palliative care issues encountered by radiation oncologists. We conducted a survey-based study of radiation oncology clinicians to define the types, frequencies, and perceived relevance of palliative care issues that arise when considering PRT for patients with advanced cancer.

\section{Methods and Materials}

Survey and participants

After receiving approval from the institutional review boards of participating radiation oncology centers we electronically surveyed radiation oncology clinicians conducting PRT consultations. We prospectively evaluated all consultations for consideration of palliative intent 
radiation therapy from May 19, 2014, to September 26, 2014, performed by a dedicated palliative radiation therapy service at a large academic cancer center and by two participating clinicians at affiliated regional hospitals. Eligible consultations included those for patients over the age of 18 with incurable, metastatic cancers. Immediately after each PRT consult, the consulting clinician was emailed a survey to be completed within 5 business days. Reminders to complete the survey were sent three times over the 5 business day interval. Fifteen radiation oncology clinicians, consisting of nurse practitioners, resident physicians, and attending physicians, were sent 162 surveys over the study period. For their participation, survey respondents received one $\$ 25$ gift card regardless of the number of surveys completed.

The survey was written and initially critiqued by three investigators (GP, VL, TB). Palliative care domains were adapted from national palliative care practice and national oncology care practice guidelines. ${ }^{10,11}$ A panel of 7 clinicians with expertise in medical oncology, radiation oncology, palliative care, and/or survey construction assessed the survey's face and content validity, ease of completion, and completion time. After incorporating each panel member's comments, we piloted the survey among radiation oncology clinicians until no further survey alterations were made after two consecutive panel members' reviews, which was achieved after 6 administrations.

Palliative care issues within eight palliative care domains were evaluated - physical symptoms, psychosocial issues, cultural consideration, spiritual needs, care coordination, advanced care planning, goals of care, and ethical and legal issues. Clinicians ranked the relevance (5-point Likert scale, 'not relevant' to 'extremely relevant') of each palliative care domain to the patient's care within radiation oncology. Additionally, clinicians indicated the perceived relevance ('yes', 'no', or 'not assessed') of 31 palliative care sub-issues related to the primary domains. Clinicians also reported whether the consulted patient's metastatic cancer diagnosis was new (within last 1 month) or established (greater than 1 month) and whether the patient, after radiation therapy consultation and intervention (where applicable), was returning to active oncological care (e.g. chemotherapy) versus to no further anti-cancer therapies (e.g., hospice care).

Study investigators extracted patient demographic (age, gender, marital status) and disease data (primary cancer type, ECOG performance status, reasons for radiation therapy 
consult, radiation therapy recommendation) from the electronic medical record corresponding to each completed survey.

\section{Statistical analysis}

The relationship between high palliative care burden ( 4 or more 'very' or 'extremely' relevant issues), diagnostic stage (new versus established metastatic diagnosis) and future care (ongoing chemotherapy vs. no further anti-cancer therapies/hospice care) was assessed with chisquare tests. Multivariable logistic regression analyses (SAS v. 9.2) examined predictors of high palliative care issue burden, including ECOG performance status, age, gender, marital status, cancer type, future care, reason for radiation therapy, and whether or not radiation therapy was ultimately recommended. Variables were entered into a multivariable model when $\mathrm{p}<0.10$ on univariable analysis and retained when $\mathrm{p}<0.05$ on multivariable analysis. Two-sided $\mathrm{p}$-values less than 0.05 were considered significant.

\section{Results}

Characteristics of the patient consultations are shown in Table 1. Over the study period, 162 patients were seen in consultation for PRT. Of the 162 surveys sent to clinicians, 140 responses were obtained ( $86 \%$ response rate). Just over half of eligible consults (56\%) involved care of a male patient. The most common primary tumor types were lung $(28 \%)$, breast $(13 \%)$, prostate (10\%), and melanoma (10\%). Pain was the leading reason for PRT consultation (57\%), followed by brain metastases (29\%). Ultimately, PRT was recommended in 120 consultations (86\%). Approximately half of patient encounters were assessed by nurse practitioners (51\%), with the remaining by resident physicians (36\%) and attending physicians (12\%).

The domains of physical symptoms (91\%), care coordination (70\%), goals of care (59\%), and psychosocial issues (52\%) were the most commonly reported domains as highly ('very' or 'extremely') relevant to care (Figure 1). When a palliative care domain was identified as relevant, clinicians further characterized the specific palliative care issues that were encountered (Table 2). Advanced care planning (29\%), cultural considerations (12\%), spiritual needs (10\%), and ethical and legal issues (7\%) were less commonly identified as being highly relevant. Most consults $(82 \%)$ had more than one palliative care domain ranked as highly relevant, and $46 \%$ of 
consults involved a high palliative care burden (Figure 2). In multivariable analyses, poor performance status (ECOG 3-4), proceeding to no further anti-cancer therapy, and a recommendation against PRT were all significantly associated with a high palliative care burden at the time of consult (Table 3).

A majority (79\%) of consults for PRT were for patients whose original diagnosis occurred more than one month prior to consultation. Of those with an established diagnosis, 88 patients $(79 \%)$ continued anti-cancer treatment and 23 patients $(21 \%)$ pursued no further anticancer therapy. Twenty-nine consultations $(21 \%)$ were performed for individuals with an original oncologic diagnosis within the past month. Nearly all of these patients began or continued anti-cancer treatment after their consultation for PRT. In two cases patients with a newly established diagnosis went on to receive hospice care following their consultation. Patients had increased rates of high palliative care burden as they moved toward no further anticancer therapies (Figure 3).

\section{Discussion}

This survey-based study demonstrates that palliative care issues are common and complex in the setting of consultations for PRT. The findings of this study contribute to the current literature by: 1) better describing the frequency and types of palliative care issues facing patients referred for PRT consultation; 2) highlighting the critical importance and relevance of generalist palliative care skills and knowledge for radiation oncology clinicians across the cancer trajectory; 3) identifying key predictors of high palliative care burden; and 4) making a case for early and systematic integration of palliative care, including consideration of hospice care, with radiation oncology care.

Importantly, this study provides valuable data to describe the types and frequency of palliative care issues that radiation oncology clinicians encounter in caring for patients with advanced cancer. The most common palliative issues - highly relevant to greater than half of PRT consultations - are management of physical symptoms, care coordination, and goals of care issues. Most PRT consultations (82\%) were found to have multiple palliative care domains relevant to the consultation. These findings combined with data from prior studies that consultations for PRT are estimated to represent at least one-third of radiation oncology 
clinicians practice, ${ }^{1,2}$ suggest that palliative care related issues constitute a substantial portion of radiation oncology practice. Furthermore, based on our study findings, patients evaluated for PRT are seen across the trajectory of their advanced cancer diagnosis. One fifth are seen at the time of an initial metastatic diagnosis, 17 percent are seen at the point of cessation of further anti-cancer therapy, and the remainder between these two time points. This highlights how radiation oncologists are involved in providing care throughout the advanced cancer continuum, and should be equipped to address generalist palliative care issues encountered throughout that trajectory.

Despite regularly encountering patients with multiple palliative care needs, radiation oncology clinicians often have insufficient training in primary palliative care. ${ }^{12}$ Palliative care physicians concur that radiation oncologists' palliative care skills are frequently lacking, with only one-third perceiving radiation oncologists as effective in managing the symptoms of radiation therapy. ${ }^{13}$ Prior studies reveal radiation oncologists discuss palliative care related topics, such as end-of-life care, less often with their patients than other clinicians. ${ }^{7,14}$ Improving radiation oncologists' comfort and competence in managing primary palliative care issues might help close gaps between recognizing the relevance of these issues and engaging patients in critical discussions regarding their management.

However, there remains a large gap between the clinical importance of palliative care competencies as suggested by this study and the academic advancement of them in the field of radiation oncology. A review of all abstracts submitted to the annual meeting of the American Society of Radiation Oncologists (ASTRO) revealed that only 4 percent of abstracts addressed the topics of symptom management or palliative care and an even smaller proportion of abstracts $(1.3 \%)$ were explicitly designated "palliative care" by their authors. ${ }^{5}$ In particular, our results reveal symptom management, care coordination, addressing goals of care, and psychosocial issues to be the most frequently encountered palliative care issues in consultations for PRT; hence, educational and academic efforts to address palliative care competencies among radiation oncologists could be focused on these topic areas.

Nearly half (46\%) of consultations for PRT were characterized by high palliative care burden, which was correlated with a poor performance status (ECOG 3 or 4), no further planned anti-cancer therapy after PRT, and a recommendation against PRT. These potential predictors may provide valuable cues to help meet the needs of patients evaluated for PRT. When one or 
more of these characteristics are present, ensuring specialty palliative care is concurrently involved would aid in addressing the complex palliative care issues these patients and their families so frequently are facing.

Furthermore, this study's description of PRT consultations with multiple, complex sets of palliative care issues raises the potential benefit of earlier and more systematic integration of specialty palliative care within radiation oncology care. Developing systems of integrating palliative care and oncologic care is consistent with the American Society of Clinical Oncology's recommendation to combine oncologic and palliative care early in the course of illness for those with metastatic disease or high symptom burden. ${ }^{15}$ Randomized trials of early palliative care in the setting of advanced cancer have shown positive effects on patient outcomes. These include increases in survival and quality of life as well as reductions in depression, use of chemotherapy at the EOL, symptom severity, and costs to the healthcare system. ${ }^{16,17,18,19}$ Efforts to integrate palliative care into radiation oncology care hold promise in improving outcomes for patients with advanced disease.

Notably, most patients in our study planned to continue active oncologic therapy following PRT, with a small minority stopping anti-cancer therapy or proceeding to hospice care. The reasons why only a minority of patients being considered for PRT engage with hospice care following their PRT is unclear. A previous study found that patients receiving PRT had a median survival of 5.6 months, ${ }^{20}$ suggesting that the majority of patients in our study would have met the eligibility criteria for hospice care. Possibilities as to why most patients receiving consultation for PRT do not proceed to hospice care include: 1) no available option to receive concurrent oncologic and hospice care; 2) underutilization of hospice care among patients with advanced cancer; 3) hesitance of clinicians, particularly radiation oncologists, to engage in discussions about EOL care ${ }^{7,14}$; and 4) underutilization of radiation therapy in patients already under hospice care. Previous studies suggest that only three percent of hospice patients receive PRT. ${ }^{21}$ The lack of sufficient hospice reimbursement to support the costs of PRT has been identified as one barrier. However, data suggest that other barriers exist; a survey of hospice professionals determined that only 52 percent feel comfortable identifying an indication for palliative intent radiation therapy despite 87 percent believing that it is an important part of the provision of hospice care. $^{21}$ 
This study has many strengths. The survey instrument underwent extensive review and iterative refinement by a multi-disciplinary group representing expertise in radiation oncology, palliative care, and survey development. The survey content was developed based on current national consensus guidelines for palliative care in oncology. ${ }^{8,10,11}$ The response rate of 86 percent was high and responding clinicians represented various levels of training, including midlevel clinicians, resident physicians, and attending physicians.

Limitations of this study include that it was conducted at a single academic center serving a single urban setting and surrounding community regions, and therefore it may not be generalizable. Furthermore, these findings reflect the perceptions of radiation oncology clinicians and hence do not directly reflect patient perspectives. Finally, this study was predominantly descriptive in nature and therefore does not suggest or evaluate the utility of any specific interventions; rather it serves as a basis for further study and hypothesis driven research regarding the evaluation and management of palliative care issues by radiation oncology clinicians.

\section{Conclusions}

Radiation oncology clinicians encounter a high volume and wide variety of palliative care issues during consultation for PRT. In the vast majority of consultations the clinicians report that palliative care issues were very or extremely relevant to the consultation. These findings highlight the need for advancing palliative care within radiation oncology through education in generalist palliative care competencies for clinicians, integration of specialty palliative care services with radiation oncology practice, and to advance palliative care within the field of radiation oncology. Such efforts will enhance generalist and specialty palliative care services provided to patients and their families frequently seen in radiation oncology and hold promise to improve their quality of life in the setting of advanced cancer. 


\section{References}

1. Janjan NA. An emerging respect for palliative care in radiation oncology. J Palliat Med. 1998;1:83-8. PubMed PMID: 15859875.

2. Tseng YD, Krishnan MS, et al. How radiation oncologists evaluate and incorporate life expectancy estimates into the treatment of palliative cancer patients: a survey-based study. Int J Radiat Oncol Biol Phys. 2013 Nov 1;87(3):471-8. doi:

10.1016/j.ijrobp.2013.06.2046

3. Guadagnolo BA, Huo J, et al. Changing trends in radiation therapy technologies in the last year of life for patients diagnosed with metastatic cancer in the US. Cancer. 2013 March 1; 119(5): 1089-1097. doi:10.1002/cncr.27835.

4. Guadagnolo BA, Liao KP, et al. Use of radiation therapy in the last 30 days of life among a large population-based cohort of elderly patients in the United States. J Clin Oncol 2013;31:80-87.

5. Jones JA, Lutz ST. Trends in Symptom Control and Palliative Care Abstracts at ASTRO 2001 to 2010. International journal of radiation oncology, biology, physics. 2011;81:645.

6. Lutz ST. A Call to Action: Hospice and Palliative Medicine Board Certification. International Journal of Radiation Oncology, Biology, Physics. 2008;72: 9-10. PubMed PMID: 18722258 .

7. Keating NL, Landrum MB, et al. Physician factors associated with discussions about endof-life care. Cancer2010;116:998-1006.

8. Institute of Medicine (IOM). Delivering High-Quality Cancer Care: Charting a New Course for a System in Crisis. Washington, DC: National Academies Press; 2013.

9. Peppercorn JM, Smith TJ, et al. American Society of Clinical Oncology statement: toward individualized care for patients with advanced cancer. J Clin Oncol. 2011; 29(6):755-60. PubMed PMID: 21263086.

10. National Comprehensive Cancer Network. NCCN Clinical Practice Guidelines in Oncology: Palliative Care. 2013.

11. National Consensus Project for Quality Palliative Care. Clinical Guidelines for Quality Palliative Care. 2013. 
12. Dharmarajan KV, Wei R, Vapiwala N. Primary Palliative Care Education in Specialty Oncology Training: More Work is Needed. JAMA Oncology 2016 2(7): 858-859.

13. McCloskey A, Tao M, et al. National survey of perspectives of palliative radiation therapy: Role, barriers, and needs. Cancer J 2007;13:130-137.

14. Mack JW, Cronin A, et al. End-of-life care discussion among patients with advanced cancer. Ann Intern Med 2012;156:204- 210.

15. Smith TJ, Temin S, et al. American Society of Clinical Oncology provisional clinical opinion: the integration of palliative care into standard oncology care. J Clin Oncol. 2012 Mar 10;30(8):880-7.

16. Temel JS, Greer JA, et al. Early palliative care for patients with metastatic non-small-cell lung cancer. N Engl J Med 2010;363:733-742.

17. Morrison RS, Dietrich J, et al. Palliative care consultation teams cut hospital costs for medicaid beneficiaries. Health Aff 2011; 30:454-463.

18. Bakitas MA, Tosteson TD, et al. Early Versus Delayed Initiation of 945 Concurrent Palliative Oncology Care: Patient Outcomes in the ENABLE III Randomized 946 Controlled Trial. J Clin Oncol 33:1438-45, 2015.

19. Zimmermann C, Swami N, et al. Early palliative care for 968 patients with advanced cancer: a cluster-randomised controlled trial. Lancet 383:1721-30, 2014.

20. Krishnan MS, Epstein-Peterson Z, et al. Predicting life expectancy in patients with metastatic cancer receiving palliative radiotherapy: the TEACHH model. Cancer. 2014 Jan 1;120(1):134-41.

21. Lutz S, Spence C, Chow E, et al. Survey on use of palliative radiotherapy in hospice care. J Clin Oncol 22:3581-3586, 2004 


\section{Tables}

Table 1: Characteristics of patients and clinicians at the time of consultation for palliative radiation therapy

\begin{tabular}{|c|c|}
\hline Characteristic & $\mathrm{N}=140$ \\
\hline \multicolumn{2}{|l|}{ Patient demographic and clinical characteristics, $n$ (\%) } \\
\hline Male & $79(56 \%)$ \\
\hline Female & $61(44 \%)$ \\
\hline Age, median (range) & $63(29-89)$ \\
\hline \multicolumn{2}{|l|}{ Cancer type } \\
\hline Lung & $39(28 \%)$ \\
\hline Prostate & $14(10 \%)$ \\
\hline Breast & $18(13 \%)$ \\
\hline Melanoma & $14(10 \%)$ \\
\hline Other & $55(39 \%)$ \\
\hline ECOG performance status, mean (sd) & $1.4(1.0)$ \\
\hline \multicolumn{2}{|l|}{ Reason for palliative radiation therapy consultation } \\
\hline Pain & $80(57 \%)$ \\
\hline Brain metastases & $41(29 \%)$ \\
\hline Impending/pathological fracture & $5(4 \%)$ \\
\hline Spinal cord compression & $18(13 \%)$ \\
\hline Tumor associated bleeding & $11(8 \%)$ \\
\hline Dyspnea & $14(10 \%)$ \\
\hline Dysphagia & $3(2 \%)$ \\
\hline Other & $8(6 \%)$ \\
\hline \multicolumn{2}{|l|}{ Radiation therapy recommendation } \\
\hline Yes & $120(86 \%)$ \\
\hline No & $20(14 \%)$ \\
\hline \multicolumn{2}{|l|}{ Future Care } \\
\hline New metastatic cancer diagnosis*/Proceeding to anti-cancer therapy & $27(19 \%)$ \\
\hline New metastatic cancer diagnosis*/No further anti-cancer therapy & $2(1 \%)$ \\
\hline Established diagnosis $\dagger /$ Continuing anti-cancer therapy & $88(63 \%)$ \\
\hline Established diagnosis $\dagger /$ No further anti-cancer therapy & $23(16 \%)$ \\
\hline \multicolumn{2}{|l|}{ Responding clinician characteristics, $\mathbf{n}(\%)$} \\
\hline Nurse practitioner & $72(51 \%)$ \\
\hline Resident physician & $51(36 \%)$ \\
\hline Attending physician & $17(12 \%)$ \\
\hline Academic practice type & $127(91 \%)$ \\
\hline Hybrid practice type: & $13(9 \%)$ \\
\hline
\end{tabular}

Abbreviations: ECOG, Eastern Cooperative Oncology Group; sd, standard deviation

* Diagnosis less than 1 month prior to consultation

$\uparrow$ Diagnosis more than 1 month prior to consultation

$\$$ Community practice affiliated with an academic center 
Table 2: Clinician reported relevance of physical, psychosocial, cultural, and spiritual issues

\begin{tabular}{|c|c|c|c|}
\hline Issue, $\mathbf{N}=\mathbf{1 4 0}$ & Yes & No & Not assessed \\
\hline \multicolumn{4}{|l|}{ Physical symptoms, n (\%) } \\
\hline Pain & $96(69 \%)$ & $44(31 \%)$ & $0(0 \%)$ \\
\hline Dysphagia & $9(6 \%)$ & $131(94 \%)$ & $0(0 \%)$ \\
\hline Bleeding & $19(14 \%)$ & $121(86 \%)$ & $0(0 \%)$ \\
\hline Non-pain neurological symptoms & $71(51 \%)$ & $69(49 \%)$ & $0(0 \%)$ \\
\hline Nausea/vomiting & $17(12 \%)$ & $123(88 \%)$ & $0(0 \%)$ \\
\hline Respiratory symptoms & $27(19 \%)$ & $113(81 \%)$ & $0(0 \%)$ \\
\hline Intestinal symptoms & $29(21 \%)$ & $110(79 \%)$ & $1(1 \%)$ \\
\hline Problems sleeping & $18(13 \%)$ & $117(84 \%)$ & $5(4 \%)$ \\
\hline Fatigue & $69(49 \%)$ & $70(50 \%)$ & $1(1 \%)$ \\
\hline \multicolumn{4}{|l|}{ Psychosocial issues, n (\%) } \\
\hline Anxiety & $47(34 \%)$ & $91(65 \%)$ & $2(1 \%)$ \\
\hline Depression & $23(16 \%)$ & $114(81 \%)$ & $3(2 \%)$ \\
\hline Family/caregiver conflict & $12(9 \%)$ & $125(89 \%)$ & $3(2 \%)$ \\
\hline Family/caregiver coping & $46(33 \%)$ & $90(64 \%)$ & $4(3 \%)$ \\
\hline Personal issues & $47(34 \%)$ & $87(62 \%)$ & $6(4 \%)$ \\
\hline \multicolumn{4}{|l|}{ Cultural issues, $\mathbf{n}(\%)$} \\
\hline \multicolumn{4}{|l|}{ Communication barrier } \\
\hline Due to language & $19(14 \%)$ & $121(86 \%)$ & N/A \\
\hline Due to beliefs/customs & $10(7 \%)$ & $130(93 \%)$ & N/A \\
\hline Spiritual needs, n (\%) & $39(28 \%)$ & $78(56 \%)$ & $23(16 \%)$ \\
\hline \multicolumn{4}{|l|}{ Care coordination issues, $\mathbf{n}(\%)$} \\
\hline \multicolumn{4}{|l|}{ Palliative care, hospice, home care discussion } \\
\hline With patient's family & $66(47 \%)$ & $74(53 \%)$ & $0(0 \%)$ \\
\hline With patient's medical care team & $54(39 \%)$ & $86(61 \%)$ & $0(0 \%)$ \\
\hline Interdisciplinary care coordination & $123(88 \%)$ & $17(12 \%)$ & $0(0 \%)$ \\
\hline Organization/participation in a family meeting & $15(11 \%)$ & $125(89 \%)$ & $0(0 \%)$ \\
\hline \multicolumn{4}{|l|}{ Advanced care planning issues, $n(\%)$} \\
\hline Living will discussion or verification & $5(4 \%)$ & $135(96 \%)$ & $0(0 \%)$ \\
\hline Power of attorney discussion or verification & $5(4 \%)$ & $135(96 \%)$ & $0(0 \%)$ \\
\hline Resuscitation preferences discussion or verification & $36(26 \%)$ & $104(74 \%)$ & $0(0 \%)$ \\
\hline \multicolumn{4}{|l|}{ Goals of care issues, $n(\%)$} \\
\hline $\begin{array}{l}\text { Consideration of prognosis in developing the } \\
\text { treatment plan }\end{array}$ & $121(86 \%)$ & $18(13 \%)$ & $1(1 \%)$ \\
\hline Discussion of prognosis with the patient or family & $80(57 \%)$ & $57(41 \%)$ & $3(2 \%)$ \\
\hline $\begin{array}{l}\text { Discussion of patient values and priorities in } \\
\text { developing the treatment plan }\end{array}$ & $99(7$ & $40(29 \%)$ & $1(1$ \\
\hline Ethical and legal issues, $n(\%)$ & $7(5 \%)$ & $133(95 \%$ & $0(0 \%)$ \\
\hline
\end{tabular}


Table 3: Regression analysis of predictors of high palliative care burden*

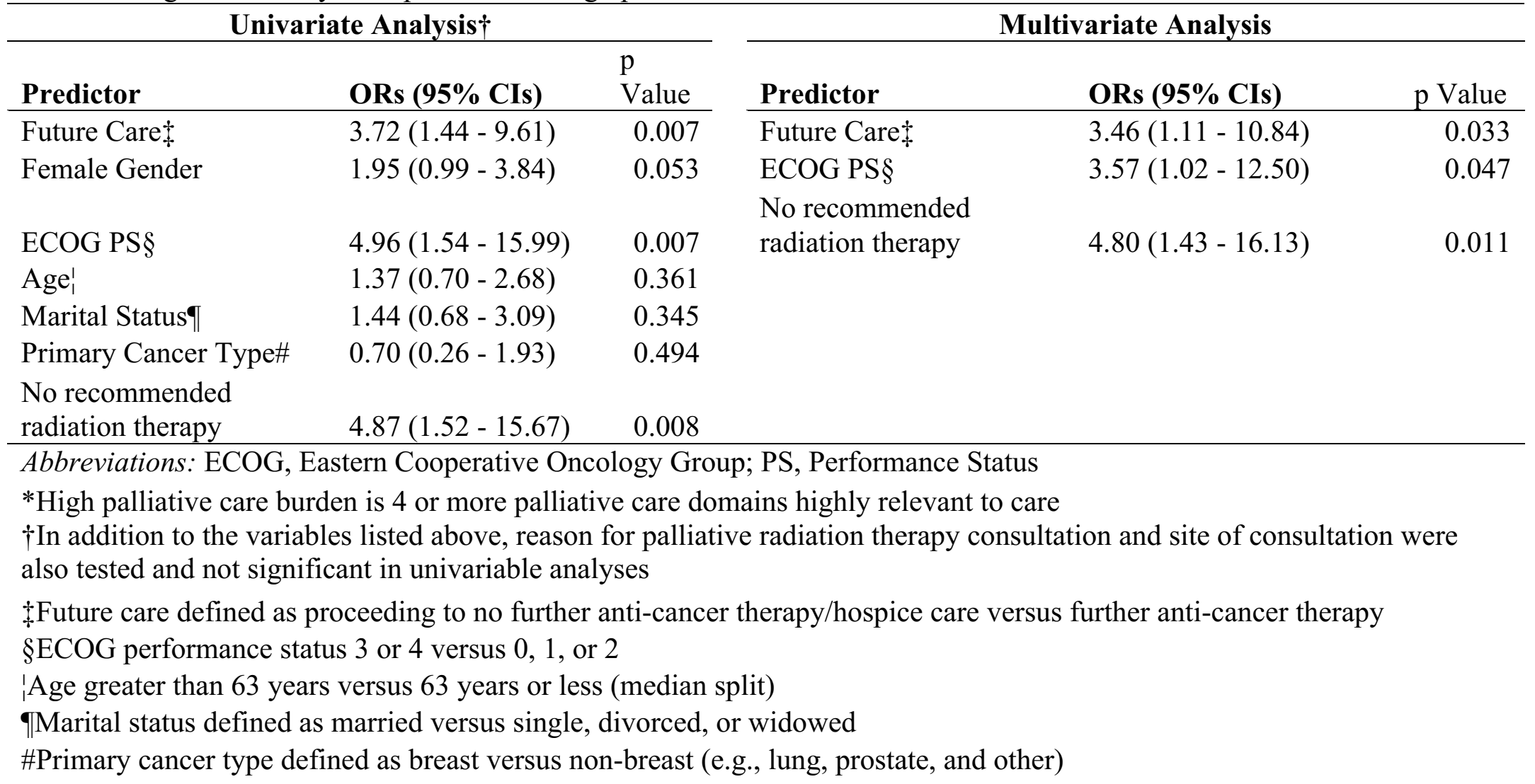




\section{Figures}

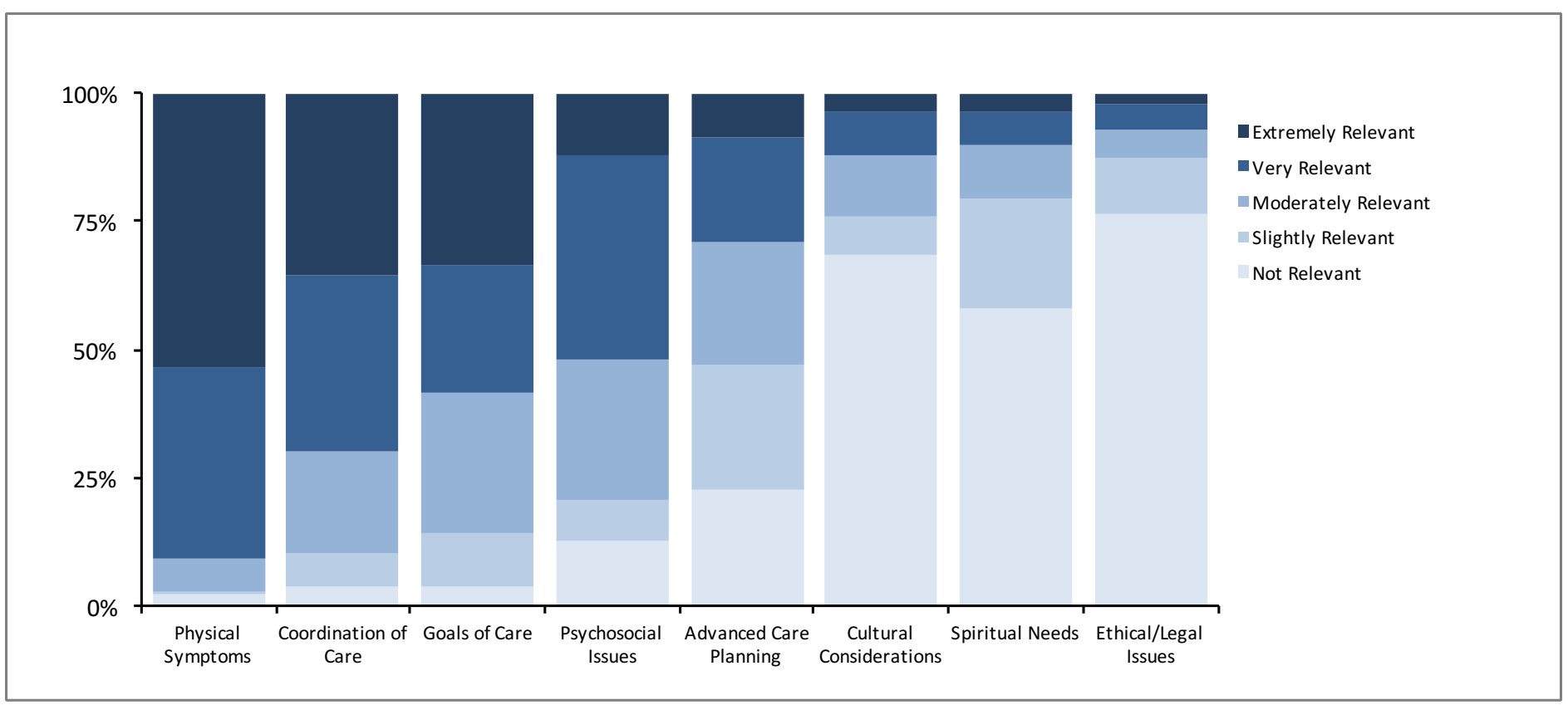

Figure 1. Relevance of palliative care domains to palliative radiation therapy consultations $(\mathrm{N}=140)$ 


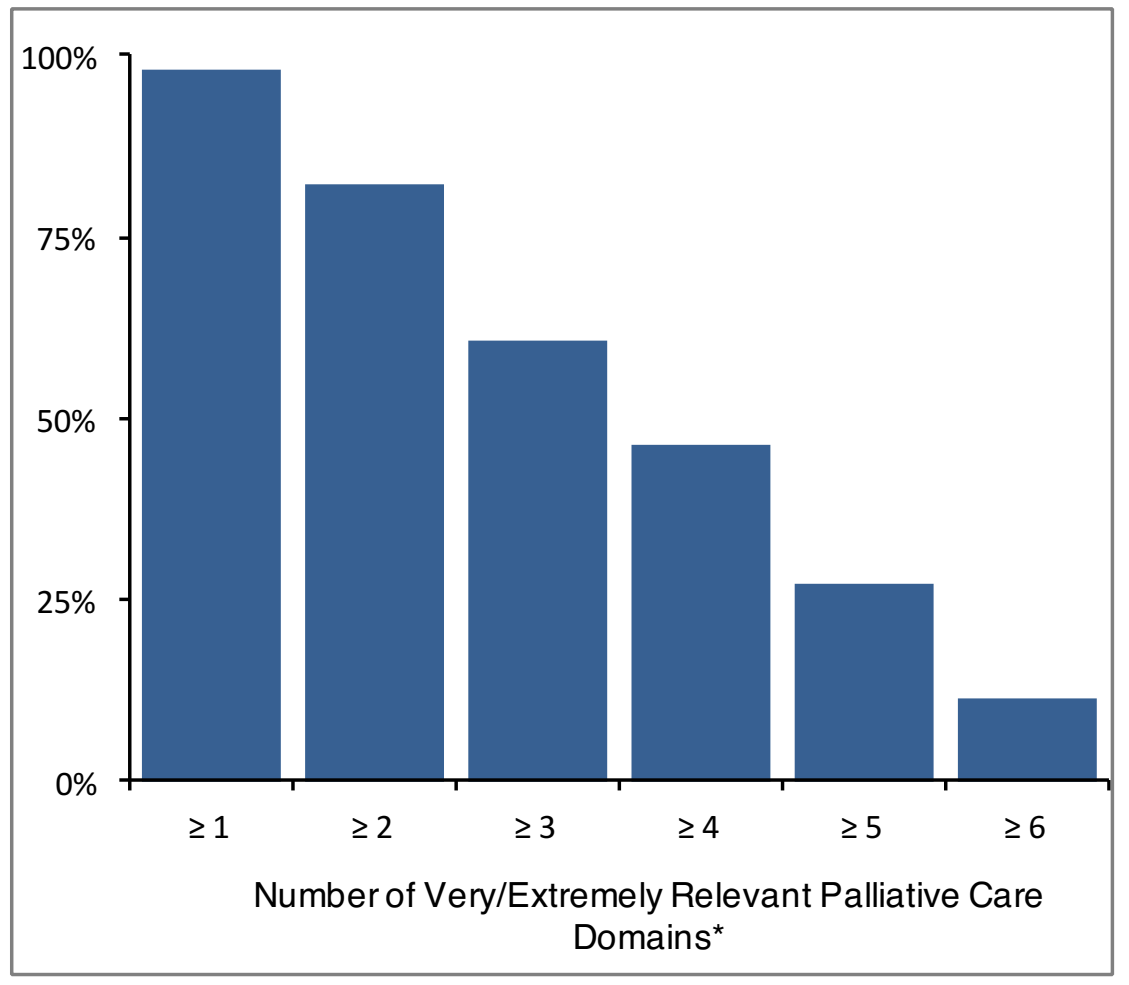

Figure 2. Cumulative palliative care burden

*Eight palliative care domains were assessed including physical symptoms, psychosocial issues, cultural issues, spiritual needs, care coordination issues, advanced care planning issues, goals of care issues, and ethical/legal issues. 


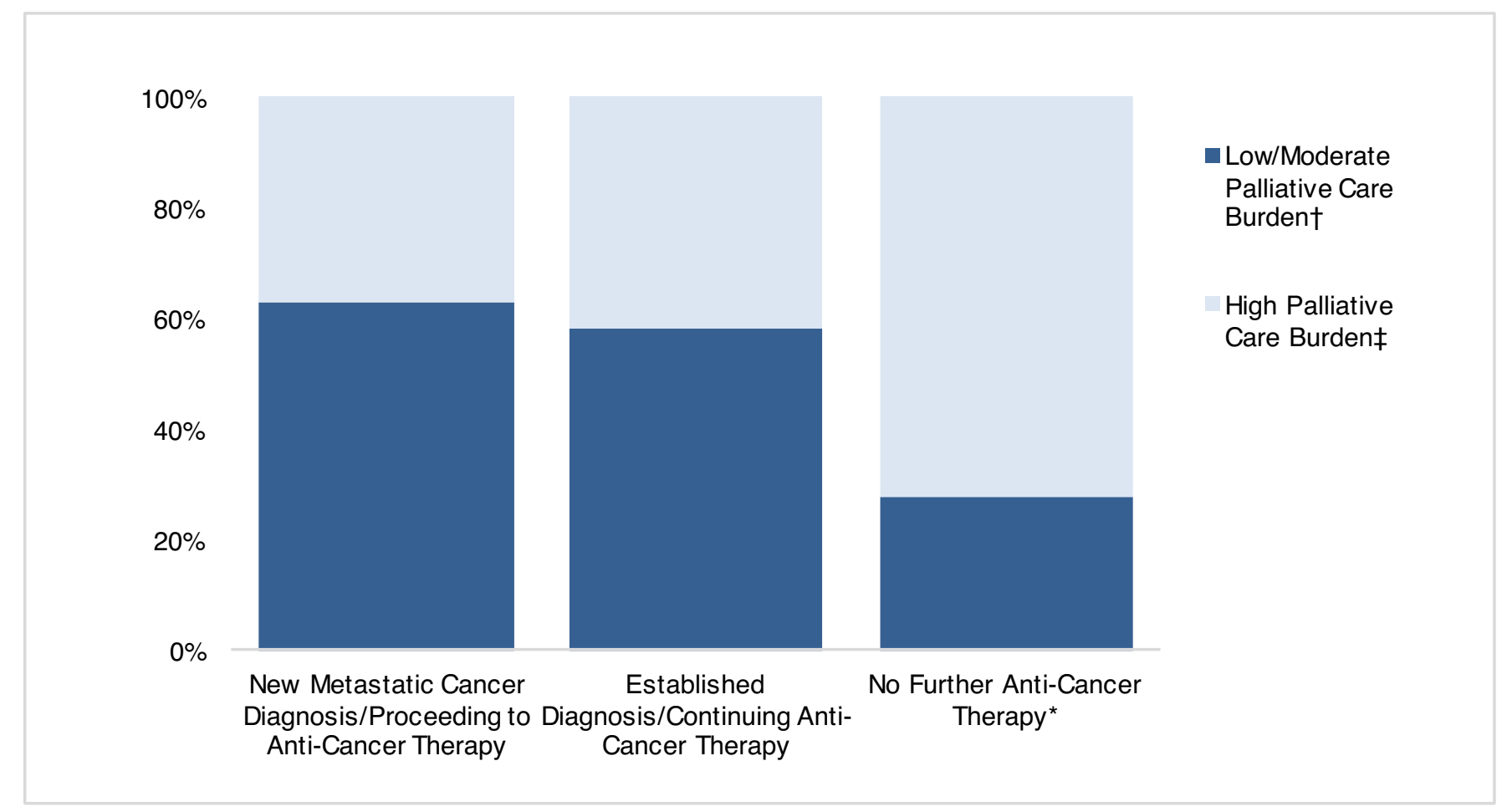

Figure 3. Future cancer therapy after radiation therapy and proportion of patients with high palliative care burden.

$* X^{2}(2, \mathrm{~N}=140)=8.21, p=0.02 . \dagger$ Low/Moderate Palliative Care Burden defined as 3 or fewer domains reported as 'very' or ‘extremely’ relevant. + High Palliative Care Burden defined as 4 or more domains reported as ‘very’ or 'extremely’ relevant. 